\title{
Cooperative Protocols Design for Wireless Ad-hoc Networks with Multi-hop Routing
}

\author{
Yuxia Lin, Joo-Han Song, and Vincent W.S. Wong \\ Department of Electrical and Computer Engineering \\ The University of British Columbia \\ Vancouver, BC, Canada \\ \{yuxial, joohans, vincentw\}@ece.ubc.ca
}

\begin{abstract}
Wireless ad-hoc networks can experience significant performance degradation under fading channels. Diversity through space has been shown to be an effective way of combating wireless fading with the multiple-input-multiple-output (MIMO) technique by transmitting correlated information through multiple antennas. The virtual MIMO technique, which allows multiple wireless stations with single antenna to form a virtual transmission array, is shown to be a viable solution from several recent studies. In this paper, we propose a complete system framework for wireless ad-hoc networks utilizing two different cooperative relaying techniques at the physical layer: the repetition coding and the space-time coding. In the data link layer, two medium access control protocols are proposed to accommodate the corresponding physical layer cooperative diversity schemes. In the network layer, diversity-aware routing protocols are proposed to determine the routing path and the relaying topology. Simulations results show significant performance gains of the proposed cooperative relaying schemes.
\end{abstract}

\section{INTRODUCTION}

Due to the impairments in the wireless channels, signal transmission through the wireless propagation medium presents various challenging problems. One of them is channel fading, which comes from the constructive and destructive interference of the multiple signal copies between the transmitter and the receiver. It can cause a wide variation in received signal's amplitude and phase over a small spatial or time interval, which degrades the signal detection performance significantly. An effective way to improve the wireless communication performance over a fading channel is the $d i$ versity technique. Instead of obtaining a single signal copy, the information symbols pass through multiple independent fading paths. A reliable communication is possible when at least one of these paths does not go into a deep fade. The probability for a transmission failure, which occurs when all the independent signal paths fade simultaneously, is thus

Permission to make digital or hard copies of all or part of this work for personal or classroom use is granted without fee provided that copies are not made or distributed for profit or commercial advantage and that copies bear this notice and the full citation on the first page. To copy otherwise, to republish, to post on servers or to redistribute to lists, requires prior specific permission and/or a fee.

QShine'08, July 28-31, 2008, Hong Kong, China .

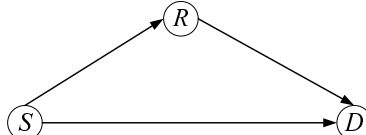

(a) Single relay scenario.

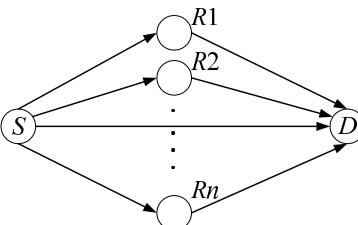

(b) Multiple relays scenario.
Figure 1: Two different cooperative diversity scenarios.

greatly reduced.

Multiple antennas can form a multiple-input multipleoutput (MIMO) system, which can provide spatial diversity gains to effectively combat fading by transmitting correlated information across the multiple antennas. To achieve the desired diversity gain, the spacing between two adjacent antenna elements should be at least on the order of the wavelength. However, many wireless devices may not meet such physical dimension requirements. But in some wireless networking scenarios, such as an ad-hoc network, it is possible to coordinate neighboring wireless nodes such that they form a virtual MIMO system and cooperatively relay each other's traffic to combat fading.

In recent years, several cooperative diversity schemes for the virtual MIMO system in wireless ad-hoc networks have been studied from the information theory and physical layer design's perspective [1-6]. The basic cooperative diversity scheme works on a single-relay channel as shown in Fig. 1(a). A source node transmits information to the destination, and a relay node first receives and then relays the information to enhance the communication between the source and destination. Some recent schemes consider two or more available relays as shown in Fig. 1(b). The performance benefits of utilizing the cooperative relaying has been well studied in the physical layer models which generally focus on the performance in regard to the outage probability and average error probabilities $[1-5]$. The mode of relay operation can be classified into two main categories: amplify-and-forward, and decode-and-forward [7]. In this paper, we use the decodeand-forward relaying which is easier to implement in the existing hardware.

With the advances in physical relay models, the medium access control (MAC) and routing protocols need to be modified in order to fully utilize the diversity gain provided by the cooperative relaying. A slotted ALOHA based cooperative MAC protocol is proposed in [8] with the decode-andforward relay. Mini-slots are used in each transmission time 
slot to accommodate the need for selection and utilization of relays. Higher throughput and lower delay performance is observed compared with the original slotted ALOHA MAC protocol.

A cooperative diversity MAC based on the 802.11 carrier sense multiple access with collision avoidance (CSMA/CA) protocol is proposed in [9]. The sender activates the cooperative relay transmission when the original 802.11 request-tosend (RTS) transmission fails. However, due to the higher reliability of the RTS transmission compared with the DATA frame transmission, this scheme neglects the case when an RTS can be successfully transmitted but the DATA frame may fail due to poor link condition. Another cooperative MAC based on 802.11 is proposed in [10] to improve the energy-saving performance of the network. The source adaptively selects the relays and assigns the transmission power to them.

Several routing schemes with cooperative considerations are also proposed recently. In [11] and [12], cooperative transmission is incorporated in route selection in wireless ad-hoc networks to improve the power consumption performance. The Co-Operative Diversity Enhanced Ad hoc Network (CODEAN) protocol is proposed in [13] by letting each intermediate node form logical clusters of relay nodes. However, these schemes mainly focus on the route selection process and assume a working MAC layer providing the services that are needed at the network layer. Some of those assumptions on the MAC layer may either be unrealistic or over-simplified.

In this paper, we propose and design a complete cooperative relay framework for wireless ad-hoc networks. For the two physical relay models considered, efficient and robust cooperative MAC protocols are proposed. They are based on the distributed carrier-sensing MAC protocols used in 802.11. This design is more robust and practical for deployment than the commonly assumed time division multiple access (TDMA) or other orthogonal MAC relaying schemes in the literature. A system framework with similar design structure as ours is proposed in [14], where a comprehensive scheme across the physical, MAC and network layers is proposed for cooperative transmission in ad-hoc networks. In their work, only space-time coding is considered at the physical layer. A primary route path is constructed using the dynamic source routing (DSR), and the relay nodes are selected at the MAC layer with an extensively modified 802.11 distributed coordination function (DCF) protocol where pilot tones are inserted after RTS frame transmission for channel estimation. Compared with [14], the proposed scheme in this paper is applicable to either repetition or space-time coding physical modules. More importantly, our relay selection is primarily carried out by the network layer with limited assistance from the MAC protocol and is based on average link signal-to-noise (SNR) estimation, so that no pilot tone is needed. Reliance on pilot tones is a major concern for protocol performance in the proposed scheme in [14], because pilot tones are assumed to be transmitted orthogonally in time where a node decides when to transmit based on its assigned globally unique ID. This introduces extra system management cost and the risk of pilot tone collision in case of mis-configuration. In our proposed scheme, the route discovery and relay selection are handled by the cooperative routing protocol (CRP). CRP is a distributed protocol and selects the relays based on the average link SNR and the two- hop neighborhood information. It considers the different bit error performance of the control and data frames when constructing the relay topology. CRP is simple to implement in the existing wireless ad-hoc networks. This design also breaks the tight coupling between MAC and network layers in [14], and can increase the reliability of the system.

The main contributions of the paper are as follows:

1. We propose two cooperative relay physical models in a slow Rayleigh fading channel with repetition or spacetime coding schemes.

2. In the data link layer, two MAC protocols are proposed for the different physical models, which have the advantages of robustness, good diversity gain, and fully distributed control.

3. In the network layer, a cooperative routing protocol (CRP) is proposed for route establishment and distributed relay selection based on link quality estimation.

4. We further propose an Enhanced CRP protocol using link SNR threshold to combat the gray zone problem which originates from the different transmission rates used by control and data frames in an ad-hoc network.

5. Simulation results show significant performance gains in regard to delay and throughput by the proposed cooperative relay design.

The rest of the paper is organized as follows. Section 2 presents the system model and our proposed cooperative MAC, and routing protocols. Section 3 presents the simulation results for performance evaluation. Conclusions and future work are presented in Section 4.

\section{SYSTEM MODEL}

We consider a wireless ad-hoc network with $N$ wireless stations. Each station has one single antenna. The physical layer model is presented in Section 2.1. Two cooperative techniques are considered at the physical layer: repetition coding with maximal ratio combining (MRC), and spacetime coding (STC). The cooperative MAC protocols proposed in Section 2.2 utilize relay nodes to assist the transmission of data traffic between source and destination nodes with cooperative relaying. For end-to-end transfer of data packets, a multi-hop routing path is determined with the CRP proposed in Section 2.3. CRP establishes a routing path and pre-selects the relay nodes. The CRP is based on the widely used ad-hoc on-demand distance vector (AODV) routing protocol [15], and may suffer from the gray zone problem [16,17], which arises from the different transmission rates for control and data frames in 802.11-based networks. An enhanced routing protocol is further proposed in Section 2.4 which uses SNR threshold to effectively combat the gray zone effect.

\subsection{Physical Layer Model}

We study a wireless ad-hoc network under a slow Rayleigh fading channel. The received instantaneous SNR $\gamma$ with fading is a random variable with the probability density function [18]:

$$
p(\gamma)=\frac{1}{\bar{\gamma}} e^{-\gamma / \bar{\gamma}}
$$


where $\bar{\gamma}$ is the average SNR. The average SNR $\bar{\gamma}$ at distance $d \geq d_{0}$ from the transmitter can be estimated by the logdistance path loss model as [19]:

$$
\bar{\gamma}=\bar{\gamma}_{0}\left(\frac{d_{0}}{d}\right)^{n}
$$

where $n$ is the path loss exponent and $\bar{\gamma}_{0}$ is the average SNR at a reference distance $d_{0}$ from the transmitter. The value of $n$ typically ranges from 2 in a free space environment to 6 in a densely populated urban environment. The value of $\bar{\gamma}_{0}$ can be calculated as:

$$
\bar{\gamma}_{0}=\frac{P_{t} G_{t} G_{r} \lambda^{2}}{(4 \pi)^{2} d_{0}^{n} L},
$$

where $P_{t}$ is the transmission power, $\lambda$ is the wavelength in meters, $L$ is the system loss factor not related to propagation, $G_{t}$ and $G_{r}$ are the transmitter and receiver antenna gains, respectively. In the rest of the paper, we choose $L=1$, $G_{t}=G_{r}=1, d_{0}=1 \mathrm{~m}$, and $n=3$.

We assume half-duplex transmission mode for cooperative relaying, where no node can receive and transmit at the same time. This is a realistic assumption because a vast majority of current radio transceivers do not have the capability of full-duplex operations on the same frequency channel. In an 802.11-based wireless network [20], there exist at least two transmission rates with different modulation and coding schemes (MCSs): the basic rate for transmitting the physical layer convergence procedure (PLCP) preamble and header, the control and broadcast frames; and the data rate for transmitting unicast data frame payloads, which is defined as the MPDU (MAC Protocol Data Unit). In this paper, we choose the basic rate to be $1 \mathrm{Mbps}$, and assume an MCS with a binary phase shift keying (BPSK) modulation with a convolutional code with Viterbi decoding. The bit error rate (BER) performance of this MCS with an instantaneous SNR $\gamma$ can be approximated using its lower bound performance [21]:

$$
P_{b}=\frac{1}{k} Q\left(\sqrt{2 d_{\text {free }}}\right),
$$

where $k$ and $d_{\text {free }}$ are the constraint length and the minimum free distance of the code, respectively. Using the popular $1 / 2$ rate convolutional code with the octal generators $133 / 171$, we have $k=7$, and $d_{\text {free }}=10$. The $Q($.$) function$ is defined as:

$$
Q(x)=\frac{1}{2 \pi} \int_{x}^{\infty} e^{-t^{2} / 2} d t .
$$

We choose the data rate to be $11 \mathrm{Mbps}$, and assume an MCS which uses a 16-ary quadrature amplitude modulation (QAM) without coding. By assuming a Gray-coded symbol assignment, the BER performance of the 16-ary QAM modulation can be approximated as [18,22]:

$$
P_{d}=1-(1-0.75 Q(\sqrt{0.8 \gamma}))^{2}
$$

Note that the assumption of using the above two MCSs does not exclude any other MCSs from being used in our proposed cooperative MAC and routing protocols. As most MCSs used in commercial products utilize more complicated physical transmission techniques, their accurate bit error performance is generally difficult to derive analytically, and sometimes may only be obtained with empirical tests and summarized in look-up tables. Our approach of using the closed-form descriptions of the bit error performance in (4) and (5) can reduce the simulation computation overhead, and facilitate the performance analysis for the cooperative MAC and routing schemes. We utilize cooperative relaying only for the data transmissions at the rate of $11 \mathrm{Mbps}$. All control and broadcast frames are transmitted in a basic rate of $1 \mathrm{Mbps}$ without cooperative relaying. One main reason for this choice is that we need the control frames to bootstrap the system by selecting the relays and establish the relaying relationship for data frame communication. Also, the basic transmission rate provides a much more robust error performance compared with the data rate.

For data transmission between source $S$ and destination $D$, two relays are selected for cooperative transmission. We denote the two chosen relays as $R_{1}$ and $R_{2}$. The mechanisms to select the relays are incorporated into the MAC and routing protocols, and will be discussed in detail in Sections 2.2 and 2.3. Here, we focus on the physical layer model of the cooperative relaying scheme. Two cooperative diversity techniques are studied:

1) Repetition coding with maximal ratio combining $(M R C)$ : After receiving the original frame from the source, the relay nodes first attempt to decode the received frame. The received frame's successful decoding depends on two factors: the preamble at the basic rate and MPDU at the data rate. Their BER can be calculated from (4) and (5), respectively. If the decoded frame is corrupted (with an incorrect cyclic redundancy check (CRC) for either the preamble or the MPDU), the relay will discard the frame. If the frame decoding is successful, the relay will transmit the frame to the destination in its assigned relay time slot. The relay transmission time for $R_{1}$ and $R_{2}$ is decided in advance with the help of the cooperative MAC protocol. It assures that two relays will not transmit the frame simultaneously to avoid a collision. After receiving the three frames from the source and the two relays, the destination $D$ will utilize MRC to combine multiple copies of the received frame, and then decode it. The BERs of the preamble header and the MPDU can be approximated as follows $[18,23]$ :

$$
\begin{gathered}
P_{p l c p}^{M R C}=\frac{1}{7} Q\left(\sqrt{20\left(\gamma_{S}+\gamma_{R_{1}}+\gamma_{R_{2}}\right)}\right), \\
P_{\text {data }}^{M R C}=1-\left(1-0.75 Q\left(\sqrt{0.8\left(\gamma_{S}+\gamma_{R_{1}}+\gamma_{R_{2}}\right)}\right)\right)^{2},
\end{gathered}
$$

where $\gamma_{S}, \gamma_{R_{1}}$ and $\gamma_{R_{2}}$ are the instantaneous SNRs of the received frames at the destination from source $S$, and relays $R_{1}, R_{2}$, respectively. This is the BER with the particular fading realization to be used in our simulations.

2) Space-time coding (STC): The two relays follow similar decode-and-forward process as in MRC. The main difference is that they will relay the frame to the destination simultaneously using a certain distributed space-time coding scheme. STC is more efficient than the repetition coding by using one less time slot to relay, but it involves more complicated signal processing at the physical layer. The receiver may successfully decode the frame from the direct transmission from the source. Failing that, the destination node can wait for the relayed STC signal in the second time slot and decode it with space-time coding. We utilize the widely used Alamouti scheme [24] on the 2 by 1 multiple-input-singleoutput (MISO) relay channel from the relays $R_{1}$ and $R_{2}$ to $D$. The SNR for the effective STC channel is the sum of 


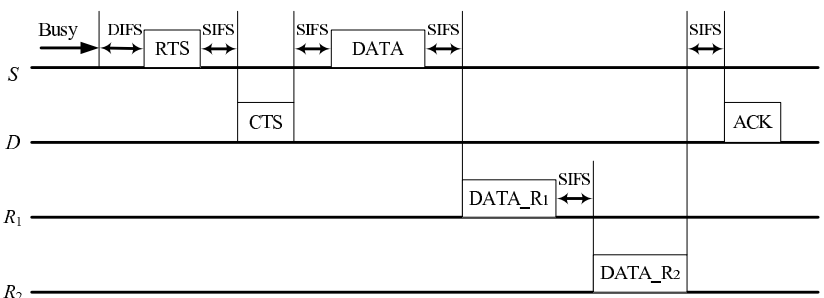

Figure 2: MRC-MAC timing sequence.

the two relay frames' SNRs [25]. As a result, the PLCP and MPDU's BER performances for STC can be derived as:

$$
P_{p l c p}^{S T C}=\frac{1}{7} Q\left(\sqrt{20\left(\gamma_{R_{1}}+\gamma_{R_{2}}\right)}\right),
$$

and

$$
P_{\text {data }}^{S T C}=1-\left(1-0.75 Q\left(\sqrt{0.8\left(\gamma_{R_{1}}+\gamma_{R_{2}}\right)}\right)\right)^{2} .
$$

\subsection{MAC Protocol Design}

We extend the CSMA/CA MAC protocol for 802.11-based WLANs to support cooperative relaying. The CSMA/CA MAC is fully distributed, and has been shown to be robust and stable.

1) $M R C-M A C$ : MRC-MAC is the MAC protocol to support repetition coding with $\mathrm{MRC}$ at the physical layer. The timing sequence of the MRC-MAC is shown in Fig. 2. The control frames RTS, clear-to-send (CTS), and acknowledgement $(\mathrm{ACK})$ are transmitted at the basic rate between a reachable pair of source and destination nodes. It is the routing layer's responsibility to maintain this reachable neighboring connection between $S$ and $D$ and selects the two relays $R_{1}, R_{2}$, which will be discussed in the next subsection. A successful RTS and CTS exchange between $S$ and $D$ leads to transmission of the DATA frame by $S$. The MAC header of the DATA frame contains four MAC addresses: the source node MAC, the destination node MAC, and two additional address fields $M A C_{-} R_{1}$ and $M A C_{-} R_{2}$, which contain the MAC address of the two relays $R_{1}$ and $R_{2}$, respectively.

When a node receives a data frame with its MAC address contained in the relay address field $M A C \_R_{1}$ or $M A C_{-} R_{2}$, it applies the decode-and-forward cooperative relaying scheme. If the relay node can successfully decode the received frame, it relays the frame to $D$ in its allocated relay time slot. For the relay node with its MAC address contained in the data frame's $M A C_{-} R_{1}$ or $M A C_{-} R_{2}$ address field, it will defer for short inter-frame space (SIFS) and DATA $+2 \times$ SIFS duration, respectively, as shown in Fig. 2. If the relay node's decoding of the data frame fails, it will remain silent during its allocated transmission time slot.

The destination node waits to receive three independent copies of the DATA frame. After the duration of $3 \times$ DATA $+4 \times$ SIFS, the destination will decode received frames with MRC. If the decoded frame successfully passes the CRC check, an ACK is sent back to the source. Otherwise, it will defer for extended inter-frame space (EIFS) and resume normal operation.

2) $S T C-M A C$ : STC-MAC is the MAC extension to support space-time coding. The timing sequence is shown in Fig. 3. Its operation is similar to the MRC-MAC, except that the two relays will both defer SIFS and transmit the relayed frame simultaneously using STC. It also employs

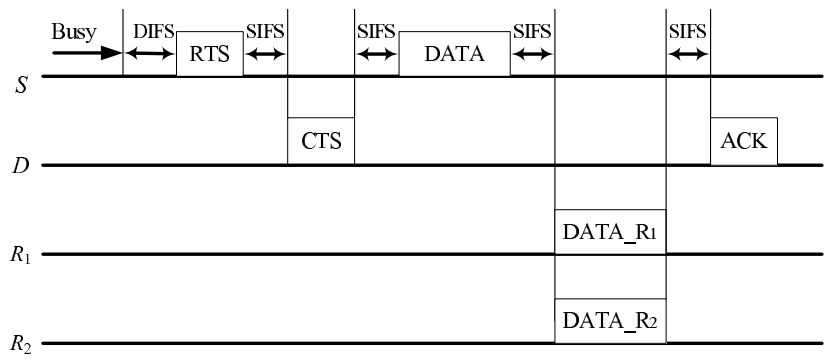

Figure 3: STC-MAC timing sequence.

decode-and-forward, which means that if a relay cannot decode the frame from $S$ correctly, it will remain silent during the relay time slot. The destination node will receive the data frame in two consecutive time slots: first, the direct transmission from the source, and then the space-time coded transmission from the two relays. If either transmission is successful, then the destination will reply with an ACK frame to the sender.

\subsection{Cooperative Routing Protocol Design}

In the MRC-MAC and STC-MAC protocols, we assume that each hop's source, destination and two relay nodes are known to the MAC layer. It is the network layer's responsibility to establish the end-to-end route, which selects each hop's source/destinations pairs and also the two relay nodes. We propose a cooperative routing protocol (CRP) for the cooperative relay network. CRP is based on the widely used AODV routing protocol in wireless ad-hoc networks. One important new feature in CRP is the discovery and maintenance of relaying nodes. To incorporate this routing feature, CRP includes two new components in the original AODV protocol: two-hop neighborhood maintenance, and relay selection.

1) Two-hop Neighborhood Maintenance: Each wireless station needs to maintain a two-hop neighborhood table which contains the MAC address of all of its immediate neighbors and those neighbors' immediate neighbors (the twohop neighborhood). Furthermore, the average link SNR between each pair of neighboring nodes need to be recorded in the two-hop neighborhood table. The HELLO message in AODV is extended to include the MAC addresses of the node's immediate neighbors and each neighbor's average link SNR information. Upon receiving a HELLO message, each node updates its two-hop neighborhood table. The receiving node adds the HELLO message's source node to its list of immediate neighbors, and the neighbor list contained in the HELLO message to the list of two-hop neighbors. The HELLO message is broadcast by each node every second.

2) Relay Selection: The end-to-end routing path is established using the route discovery mechanisms of AODV with the route request and route reply (RREQ/RREP) control frames. We expand the routing table with two fields $R_{1}$ and $R_{2}$, which contain the MAC addresses of the two selected relay nodes for the next hop transmission. Whenever a new route entry is inserted into the routing table which provides a route entry from node $i$ to destination $D$ with the next hop $j$, the two relay nodes $R_{1}$ and $R_{2}$ are selected based on $i$ 's two-hop neighborhood table and inserted into the route entry. The algorithm to select the two relay nodes is as follows: 
1. For every direct neighbor $x$ of node $i$, look up $x$ 's list of direct neighbors $L_{x}$, which is contained in $i$ 's two-hop neighborhood table.

2. If the next hop node $j \in L_{x}$, then determine the minimum SNR between the two logical links $(x, i)$ and $(x, j)$ :

$$
\eta_{x}=\min [S N R(x, i), S N R(x, j)]
$$

3. Sort $\eta_{x}$ for all $x$. The two nodes associated with the two highest $\eta_{x}$ values are selected as $R_{1}$ and $R_{2}$, respectively.

This algorithm effectively chooses two nodes which are common immediate neighbors of both $i$ and $j$, and having the two highest of the minimum SNR of the relay channels (from $i$ to relay, and relay to $j$ ). This effectively avoids selecting relays which have a weak link with either the sender $i$ or the receiver $j$.

\subsection{Enhanced CRP}

The CRP protocol proposed in the previous section can enhance the wireless communcation compared with the original AODV. However, the CRP protocol still suffers from the well-known gray zone problem of AODV in an 802.11 network $[16,17]$. This phenomenon stems from the large difference in BER performance between MCSs used by the basic rate and data rate transmission. The AODV HELLO and RREQ packets are broadcast at the basic rate which has a much more robust BER performance than the higher rate of data transmissions. The difference in control frame and data frame's length (a few hundred bits versus thousands of bits) further exaggerates the frame error rate of the control and data frames. This creates long but unreliable routing hops in the gray zone for transmitting large data frames.

Various methods can be utilized to combat the effects of gray zone [26]. In this paper, we propose an enhanced CRP protocol (E-CRP) by using a threshold-based approach to mitigate the gray zone effect. When a node maintains its two-hop neighborhood table, the received HELLO packet's average SNR is recorded with a moving average. The HELLO packet's source node will be added as an immediate neighbor in the two-hop neighborhood table only when the average SNR of the link is above a threshold value $\gamma_{0}$. All received AODV control broadcast packets will be subject to the filtering by the neighborhood table. A broadcast packet will be dropped if its source is not recorded as the receiving node's immediate neighbor despite a possible successful reception. The appropriate $\gamma_{0}$ threshold should be set such that it provides a well connected network topology and also maintains the average SNR of the link to be above reasonable levels. In this paper, a practical $\gamma_{0}$ value is selected based on simulation experiments.

\section{PERFORMANCE EVALUATION}

Simulation experiments are carried out by using the ns-2 simulator. The following new modules are implemented in the ns-2 simulator: the fading channel and the physical error model, the cooperative MAC and routing protocols proposed in Section 2. The system parameters used in the simulation are listed in Table 1.
Table 1: Simulation Parameters

\begin{tabular}{|l|c|}
\hline \hline Basic Rate & $1 \mathrm{Mbps}$ \\
\hline Data Rate & $11 \mathrm{Mbps}$ \\
\hline Transmission Power & 31 \\
\hline Minimum Contention Window Size & 1023 \\
\hline Maximum Contention Window Size & $192 \mathrm{bits}$ \\
\hline DSSS PLCP Preamble and Header & $192 \mathrm{bits}$ \\
\hline MAC Header & $32 \mathrm{bits}$ \\
\hline FCS (Frame Check Sequence) & $304 \mathrm{bits}$ \\
\hline ACK Frame Size (include Headers) & $1000 \mathrm{bytes}$ \\
\hline CBR Data Packet Size & $20 \mu \mathrm{s}$ \\
\hline Time Slot & $10 \mu \mathrm{s}$ \\
\hline SIFS & $50 \mu \mathrm{s}$ \\
\hline DIFS & 4 \\
\hline Max Number of Retransmissions & TCP Reno \\
\hline TCP Source Protocol Version & TCP SACK \\
\hline TCP Sink Protocol Version & \\
\hline
\end{tabular}

\subsection{Linear Topology Test}

A linear topology with 10 nodes forming a 3-hop route between the source $S$ and destination $D$ is shown in Fig. 4. In this test, the route and relay nodes are known. As a result, it only tests the performance of cooperative MAC protocols. A 100 kbps constant bit rate (CBR) traffic flow is transmitted from $S$ to $D$. The distance between two adjacent nodes is $d$, and $d$ varies from $50 \mathrm{~m}$ to $160 \mathrm{~m}$. Under our proposed cooperative relaying scheme, two relays are used in each hop with the MRC-MAC or the STC-MAC. The packet delivery ratio of the network under cooperative relaying is compared with the original 802.11 MAC where no relays are utilized. The results for the CBR packet delivery ratio and the end-toend delay are shown in Fig. 5 (a) and (b), respectively. We can see that MRC-MAC and STC-MAC consistently achieve better performance than the 802.11 MAC. The cooperative MACs maintains the delivery ratio above $99 \%$ while the hop distance increases to $140 \mathrm{~m}$. However, with $d$ increasing further, the delivery ratio begins to drop significantly, which means that the link is becoming too unreliable even for cooperative schemes to maintain a low BER on the link. This indicates the start of the gray zone area where a low rate control frames may still have high enough success rate, but the data frame's error rate has significantly increased.

Simulations tests with one TCP flow from $S$ to $D$ is carried out, and the throughput performance under the three different MAC schemes is shown in Fig. 6. The 802.11 MAC has better performance when the distance is shorter than $80 \mathrm{~m}$. This is because within such a short distance, the link is robust enough that the frame error rate is already small without cooperation. Within this short range, cooperative MAC simply consumes more transmission time slots and lead to poorer performance. But when $d$ increases further, the cooperative relaying's effectiveness begins to show up. These results can help us to decide what range of hop distance is optimal for cooperative routing protocols.

In all the tests, the STC-MAC has both a delay performance gain for CBR traffic and a throughput gain for TCP traffic compared with MRC-MAC because it utilizes one less time slot for cooperative transmission. But STC-MAC requires more enhanced signal processing capabilities in wireless stations. 


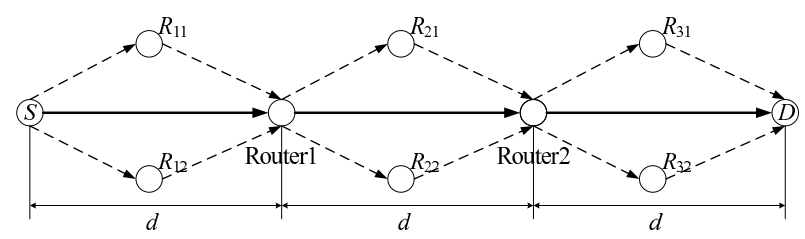

Figure 4: Linear topology.

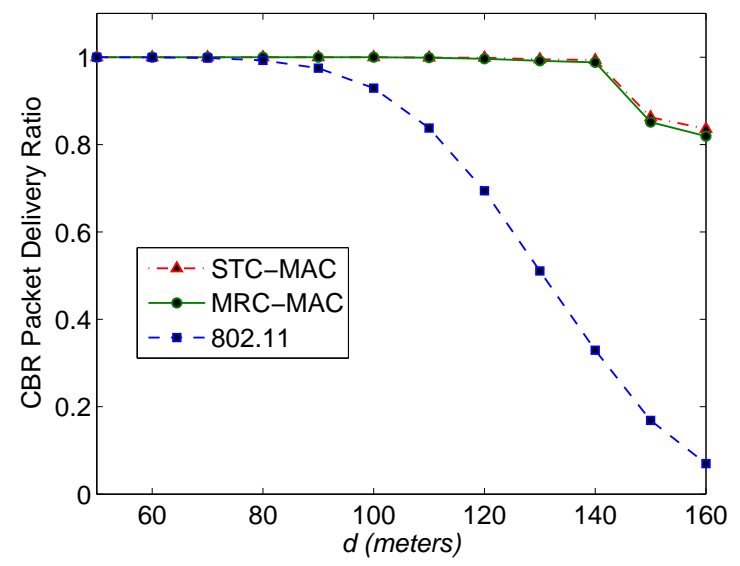

(a) Packet delivery ratio for CBR traffic.

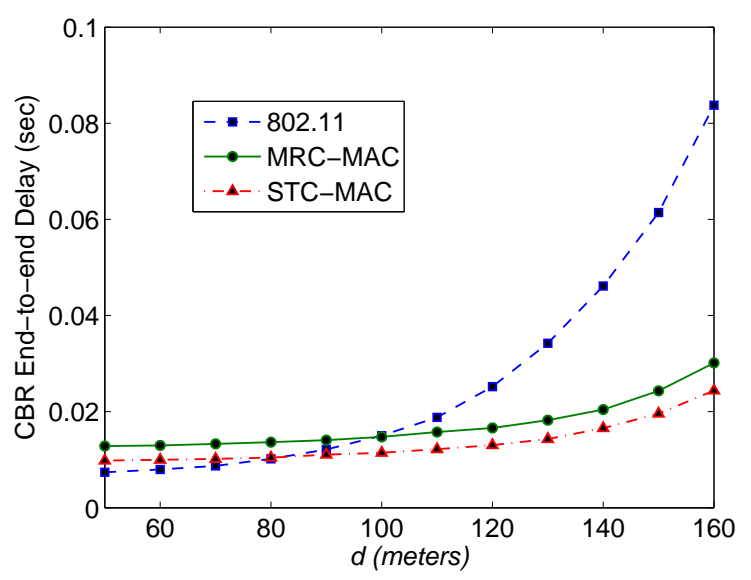

(b) End-to-end delay for CBR traffic.

Figure 5: Linear topology tests.

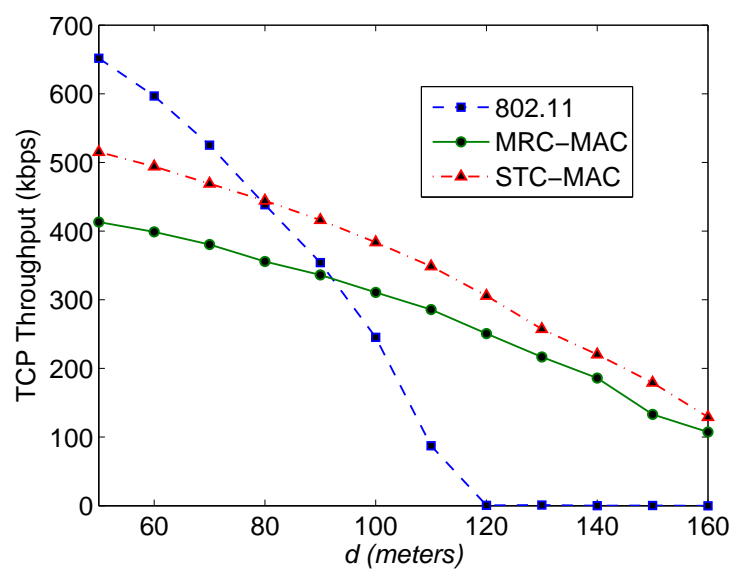

Figure 6: TCP throughput with linear topology.

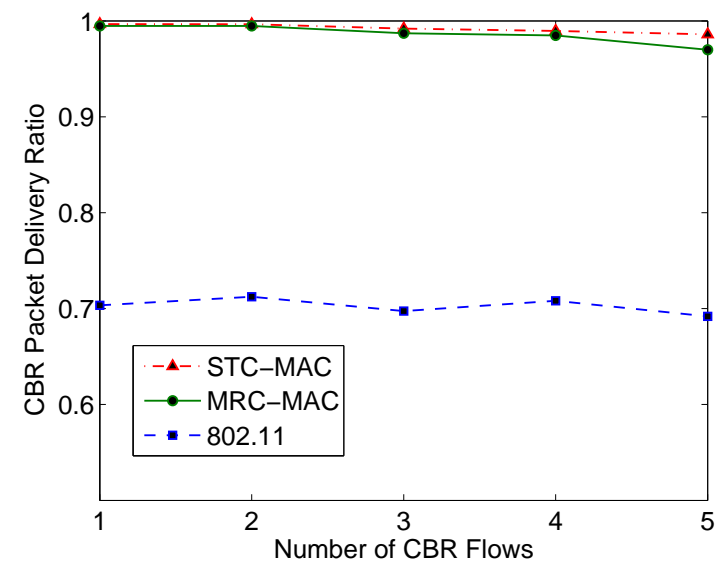

(a) Packet delivery ratio for CBR traffic.

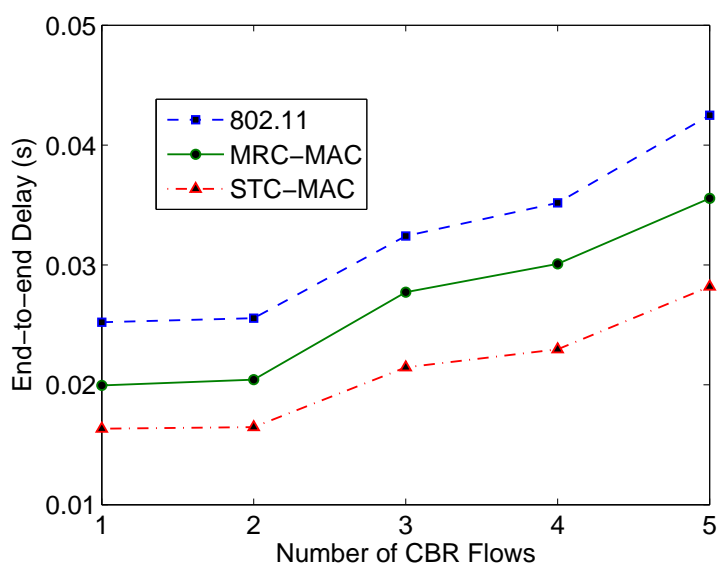

(b) End-to-end delay for CBR traffic.

Figure 7: Random topology tests with E-CRP.

\subsection{Random Topology Test}

To fully test the CRP protocol, we carry out a random topology test in a $500 \mathrm{~m} \times 500 \mathrm{~m}$ square area. 50 wireless nodes are randomly located within the area. One to five $50 \mathrm{kbps}$ CBR flows exist in the network with each flow's source and destination randomly selected. We compare the CBR traffic's performance under E-CPR/cooperative MAC with AODV/802.11 MAC. The results for CPR proposed in Section 2.3 without the gray zone enhancement showed a performance decline of around $20 \%$ to $30 \%$ compared with E-CPR. Due to the restraint on space, only E-CPR's results are presented. To have a fair comparison, we also applies the SNR threshold policy to the original AODV to obtain an enhanced AODV protocol for gray zone reduction. $\gamma_{0}$ is chosen to be $10 \mathrm{~dB}$ which corresponds to limiting the perhop distance to approximately $140 \mathrm{~m}$ from (2). This is the distance where the gray zone effects begin to show up from Figs. 5 and 6 in the linear topology tests.

The CBR packet's delivery ratio and end-to-end delay are shown in Fig. 7 (a) and (b). The delivery and delay performance slightly declines with increasing traffic load, but the cooperative MAC/routing schemes consistently maintain a much better performance than 802.11 MAC with AODV. STC-MAC also out-performs MRC-MAC with the 
gains from its more enhanced signal processing capabilities.

\section{CONCLUSIONS}

In this paper, we proposed a complete system design for utilizing cooperative relaying in wireless ad-hoc networks to improve the network performance. In the data link layer, we proposed the MRC-MAC and STC-MAC protocols when the underlying physical layer uses the repetition coding with maximal ratio combining and space-time coding, respectively. In the network layer, we proposed the CRP and E-CRP protocols for routing and relay selection. Simulation results from the linear and random topologies show significant performance gains from the E-CRP with the two cooperative MAC protocols.

For future work, we plan to further improve the cooperative system design by adaptively deciding whether to invoke cooperative relaying on each hop. We will also study the cooperative relaying system with analytical models and design algorithms for optimizing system operation parameters such as the SNR threshold for E-CRP.

\section{Acknowledgment}

This work was supported by the Natural Sciences and Engineering Research Council of Canada (NSERC).

\section{REFERENCES}

[1] A. Sendonaris, E. Erkip, and B. Aazhang, "User cooperation diversity - Part I: System description," IEEE Trans. Commun., vol. 51, no. 11, pp. 1927-1938, Nov. 2003.

[2] — - "User cooperation diversity - Part II: Implementation aspects and performance analysis," IEEE Trans. Commun., vol. 51, no. 11, pp. 1939-1948, Nov. 2003.

[3] P. A. Anghel and M. Kaveh, "Exact symbol error probability of a cooperative network in a Rayleigh-fading environment," IEEE Trans. Wireless Commun., vol. 3, no. 5, pp. 1416-1421, 2004.

[4] J. Hu and N. C. Beaulieu, "Closed-form expressions for the outage and error probabilities of decode-and-forward relaying in dissimilar Rayleigh fading channels," in Proc. of IEEE ICC, Glasgow, Scotland, June 2007.

[5] G. Scutari and S. Barbarossa, "Distributed space-time coding for regenerative relay networks," IEEE Trans. Wireless Commun., vol. 4, no. 5, pp. 2387-2399, 2005.

[6] S. Vakil and B. Liang, "Effect of joint cooperation and multi-hopping on the capacity of wireless networks," in Proc. of IEEE SECON, San Francisco, CA, June 2008.

[7] J. N. Laneman, D. N. C. Tse, and G. W. Wornell, "Cooperative diversity in wireless networks: Efficient protocols and outage behavior," IEEE Trans. Inform. Theory, vol. 50, no. 12, pp. 3062-3080, Dec. 2004.

[8] J. M. Shea, T. F. Wong, and W.-H. Wong, "Cooperative-diversity slotted ALOHA," Wireless networks, vol. 13, no. 3, pp. 361-369, July 2006.

[9] S. Moh, C. Yu, S.-M. Park, H.-N. Kim, and J. Park, "CD-MAC: Cooperative diversity MAC for robust communication in wireless ad hoc networks," in Proc. of IEEE ICC, Glasgow, Scotland, June 2007.
[10] A. Azgin, Y. Altunbasak, and G. AlRegib, "Cooperative MAC and routing protocols for wireless ad hoc networks," in Proc. of IEEE Globecom, St. Louis, MO, Nov. 2005.

[11] X. Fang, T. Hui, Z. Ping, and Y. Ning, "Cooperative routing strategies in ad hoc networks," in Proc. of IEEE VTC-Spring, Stockholm, Sweden, May 2005.

[12] N. Kim, B. An, D. Kim, and Y. Lee, "Wireless ad-hoc networks using cooperative diversity-based routing in fading channel," in Proc. of IEEE PACRIM, Victoria, Canada, Aug. 2007.

[13] M. A. Tope, J. C. McEachen, and A. C. Kinney, "Routing performance of cooperative diversity in ad-hoc networks," in Proc. of IEEE ISCC, Pula-Cagliari, Italy, June 2006.

[14] G. Jakllari, S. V. Krishnamurthy, M. Faloutsos, P. V. Krishnamurthy, and O. Ercetin, "A cross-layer framework for exploiting virtual MISO links in mobile ad hoc networks," IEEE Trans. Mobile Comput., vol. 6, no. 6, pp. 579-594, June 2007.

[15] C. Perkins, E. Belding-Royer, and S. Das, "Ad hoc on-demand distance vector (AODV) routing," IETF-RFC 3561, 2003.

[16] H. Lundgren, E. Nordstrom, and C. Tschudin, "The gray zone problem in IEEE $802.11 \mathrm{~b}$ based ad hoc networks," ACM Mobile Comput. and Comm. Review, vol. 6, no. 1, pp. 104-105, July 2002.

[17] W. Kim, J. Lee, T. Kwon, S.-J. Lee, and Y. Choi, "Quantifying the interference gray zone in wireless networks: A measurement study," in Proc. of IEEE ICC, Glasgow, Scotland, June 2007.

[18] J. G. Proakis, Digital Communications, 4th ed. McGraw Hill Higher Education, 2000.

[19] T. S. Rappaport, Wireless Communications: Principles and Practice, 1st ed. Prentice Hall, 1996.

[20] IEEE 802.11 WG, "IEEE standard: Wireless LAN medium access control (MAC) and physical layer (PHY) specifications," 2003.

[21] S. B. Wicker, Error Control Systems for Digital Communication and Storage, 1st ed. Prentice Hall, 1995.

[22] S. Maghrebi, M. Lotfizad, and M. Ghanbari, "The better performance of the new non-rectangular QAM with FHT in ADSL system based on DMT without cyclic prefix," in Proc. of Int'l. Conference on Digital Signal Processing, Cardiff, Wales, UK, July 2007.

[23] A. Ribeiro, X. Cai, and G. B. Giannakis, "Symbol error probabilities for general cooperative links," IEEE Trans. Wireless Commun., vol. 4, no. 3, pp. 1264-1273, May 2005.

[24] S. Alamouti, "A simple transmit diversity technique for wireless communications," IEEE J. Select. Areas Commun., vol. 16, no. 8, pp. 1451-1458, Oct. 1998.

[25] R. N. A. Paulraj and D. Gore, Introduction to Space-time Wireless Communications, 1st ed. Cambridge, 2003.

[26] B. Awerbuch, D. Holmer, and H. Rubens, "The medium time metric: high throughput route selection in multi-rate ad hoc wireless networks," ACM/Springer Mobile Networks and Applications, vol. 11, no. 2, pp. 253-266, Apr. 2006. 